\title{
Syndrome aigu d'irradiation : les atteintes du système gastro-intestinal
}

\author{
N.M. GRIFFITHS ${ }^{\prime}$, I. DUBLINEAU ${ }^{\prime}$, F. LEBRUN $^{\prime}$, C. LINARD ${ }^{\prime}$, P. MONTI $^{\prime}$,

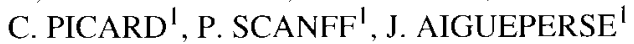

(Manuscrit reçu le 30 mars 2001 , accepté le 29 juin 2001)

RÉSUMÉ L'exposition aux rayonnements ionisants à des doses moyennes ou fortes entraîne une pathologie mixte. Dans plusieurs accidents d'irradiation, il a été constaté que les dysfonctionnements intestinaux étaient fortement impliqués dans le décès des patients. Cependant, l'apparition du syndrome gastro-intestinal, classiquement défini comme étant la perte de la muqueuse intestinale, n'est jamais très évidente. Néanmoins, il est admis que l'atteinte du système gastro-intestinal, plus particulièrement sa fonction de barrière, joue un rôle important dans l'évolution du syndrome de défaillance multiviscérale (Multiple Organ Failure) observée dans les derniers accidents (Tokaï Mura). Une exposition aux rayonnements ionisants provoque des modifications de la motricité intestinale qui peuvent conduire à la diarrhée. La diarrhée peut provenir également des altérations des processus d'absorption et/ou de sécrétion. En effet, une perte des cellules de la barrière intestinale et des modifications concomitantes de la fonction des systèmes de transport des nutriments et/ou des électrolytes sont observés. Ces fonctions intestinales sont sous le contrôle des multiples agents (tels que des contenus intestinaux, des neurotransmetteurs, des agents paracrines et des hormones) qui sont aussi modifiés. À l'heure actuelle, les traitements contre les atteintes digestives restent symptomatiques mais l'utilisation des facteurs de croissance et/ou l'apport de nouvelles cellules semblent prometteur pour l'avenir.

ABSTRACT Acute syndrome of radiation: injuries to the gastrointestinal tract.

Exposure to ionising radiation at medium to high doses results in the manifestation of mixed pathologies. Following the analysis of several radiation accidents it is clear that intestinal injury influences patient survival. However the appearance of the classically defined gastrointestinal syndrome is not always evident. Nevertheless injury to the gastrointestinal tract, in particular loss of barrier function, seems to play an important role in the development of Multiple Organ Failure such as reported in the recent accident at Tokai Mura. Ionising radiation overexposure results in changes in intestinal motility and nutrient, fluid and electrolyte absorption and secretion all which may contribute to the genesis of diarrhoea. In addition to modified cellular transport properties for nutrients or electrolytes, important loss of epithelial cells is also a major contributing factor. Intestinal functions are controlled by many factors such as neurotransmitters, locally released mediators from endocrine cells or immunocompetent cells in addition to luminal agents. To date, treatment of radiation-induced gastrointestinal injury is mainly symptomatic. However treatments such as growth factors, anti-inflammatory cytokines as well as cellular transplantation remain to be validated in the radiation accident situation.

\footnotetext{
Institul de radioprotection et de sûreté nucléaire, DPHD/SARAM, BP 17, 92262 Fontenay-aux-Roses, France.
} 


\section{Introduction}

Une exposition aux rayonnements ionisants à des doses moyennes ou fortes, entraîne une pathologie mixte, avec notamment des atteintes digestives qui peuvent contribuer fortement à la mort d'un individu, accidentellement irradié. Chez les accidentés les plus irradiés de Tchernobyl, le syndrome gastro-intestinal a participé à la mort dans au moins 11 cas (Baranov et Guskova, 1990). La plupart de ces personnes ont déclaré une diarrhée liquide et hémorragique plusieurs jours avant leur décès (Moscow-Ulm Radiation Accident Clinical History Data Base). Dans le cas de l'accidenté de Soreq (Israël, 1990), le patient a eu des épisodes de diarrhée profuse et hémorragique ; l'intestin grêle et le gros intestin ont été très endommagés (IAEA, 1993). L'autopsie a révélé des modifications profondes de la morphologie intestinale caractérisées par la disparition complète des cryptes associée à une dénudation de la muqueuse. Parallèlement, la morphologie de la muqueuse et de la sous-muqueuse colique a été très modifiée. De plus, des modifications profondes des éléments vasculaires tels que des adèmes et occasionnellement des thromboses ont été observées. Néanmoins, la cause de la mort a été attribuée à une défaillance respiratoire associée à une réaction probable contre la greffe de moelle osseuse qui avait été faite. Il est important de noter que le système digestif est souvent l'organe cible des effets secondaires néfastes d'une greffe de moelle osseuse (Hill et al., 1997).

Des atteintes digestives après les expositions aux rayonnements ionisants ont également été rapportées pour les accidents de Goiânia (Brésil, 1987 (AEA, 1988)) et de Nesvizh (Biélorussie, 1991 (IAEA, 1996)). Des problèmes digestifs ont en effet été rencontrés chez les irradiés de Goiânia dont huit ont exprimé une diarrhée profuse pendant la phase prodromale. Chez quatre personnes, des hémorragies intestinales ont été retrouvées à l'autopsie. L'accidenté de Nesvizh a exprimé un syndrome prodromal comprenant vomissements et diarrhées. Ce patient, qui n'a pas été greffé, a survécu 107 jours. L'autopsie a montré que l'estomac était rempli de sang, l'iléon était cyanotique et hémorragique avec une perte de muqueuse sur $50 \mathrm{~cm}$. Le reste du tractus digestif n'était pas significativement endommagé, ce qui a suggéré une récupération partielle de cet organe (IAEA, 1996). La sévérité des atteintes intestinales peut dépendre de l'étendue de la zone exposée car il a été observé que le volume de l'intestin irradié (chez des patients soumis à la radiothérapie) joue un rôle important dans l'apparition de la diarrhée (Letschert et al., 1994).

Les effets de l'irradiation sur le système gastro-intestinal peuvent être classés par les symptômes observés et par leur temps d'apparition après l'exposition aux rayonnements. Pendant la phase aiguë ou prodromale des vomissements, des nausées, un ralentissement de la vidange gastrique et de fortes diarrhées peuvent 
se manifester. L'apparition de ces symptômes dits « précoces » est d'autant plus rapide que la dose est forte. Elle dépend également du débit de dose, de la nature du rayonnement et de l'étendue des champs irradiés. L'apparition des diarrhées, très précoces, est souvent le signe d'un mauvais pronostic pour l'individu car elle est associée à des dommages très sévères et fatals (Spitzer, 1995). Cette diarrhée est probablement liée à des facteurs neuro-hormonaux et à des changements de la motricité intestinale. La mise en place de la phase d'état du syndrome gastrointestinal est caractérisée par une diarrhée rebelle, profuse et hémorragique associée à la perte de la muqueuse intestinale. D'autres signes cliniques de cette phase sont les vomissements, les diarrhées, les crampes et les douleurs abdominales.

Le système gastro-intestinal est particulièrement sensible aux rayonnements ionisants en raison du renouvellement constant de l'épithélium à partir des cellules souches. Les atteintes gastro-intestinales sont la conséquence d'une atténuation de la production des cellules clonogéniques retrouvées dans les cryptes de Lieberkühn (Hendry et al., 1983 ; Potten, 1990). Le temps de latence existant avant l'apparition du syndrome gastro-intestinal dépend de la durée de vie et de la migration des cellules différenciées vers les villosités ou la surface colique car ces cellules sont moins affectées par l'irradiation que les cellules souches. Quastler en 1956 a défini le syndrome gastro-intestinal comme correspondant à une perte de $80 \%$ de la muqueuse de l'intestin grêle (Quastler, 1956). Ce syndrome gastrointestinal est associé à des pertes hydrominérales et protéiques, à des infections et à des hémorragies. Récemment, il a été montré qu'un des facteurs importants dans le développement du SGI est la microcirculation intestinale, plus particulièrement, l'apoptose des cellules endothéliales (Paris et al., 2001). Cette étude montre que chez la souris pour des doses élevées ( 15 Gy irradiation corps entier) l'apoptose des cellules endothéliales est la lésion primaire conduisant à la mort cellulaire des unités crypte-villosité. Cependant, à 14 Gy les atteintes des cellules épithéliales ont été moins marquées et concomitantes à un niveau d'apoptose moindre au niveau des cellules endothéliales. Cette étude a une importance capitale vis-à-vis du dogme de la mort intestinale où la perte des cellules souches représente un élément clé dans le développement du syndrome gastrointestinal.

L'implication des atteintes gastro-intestinales dans les problèmes hématopoïétiques ou dans les atteintes d'autres organes reste à définir. Dans un certain nombre d'accidents (Tchernobyl, Tokaï Mura) les décès ont été attribués à des pathologies mixtes conduisant au «syndrome de défaillance multiviscérale » (Multiple Organ Failure). Ainsi, d'une part les atteintes gastro-intestinales peuvent avoir des conséquences sur le système hématopoïétique et sur d'autres organes, et d'autre part, les atteintes d'autres organes peuvent modifier le fonctionnement intestinal. Pour les doses les plus fortes, il a été difficile de déterminer les causes exactes de 
la mort et en particulier si elle était due aux effets de l'irradiation sur la peau et sur l'intestin (Baranov, 1994). Des brûlures très graves, par exemple, peuvent provoquer une ischémie intestinale, une perte de la barrière intestinale et par la suite une translocation bactérienne (Maejima et al., 1984 ; Deitch et al., 1989 ; Moore, 1999).

\section{Dysfonctionnements gastro-intestinaux radio-induits}

Une exposition à une dose n'entraînant pas le syndrome gastro-intestinal peut provoquer des dysfonctionnements gastro-intestinaux sans mettre directement en danger la vie de l'individu. Ces dysfonctionnements peuvent être associés à des altérations de la barrière épithéliale (plus particulièrement les processus d'absorption et/ou de sécrétion), de la motricité de l'intestin grêle et/ou du côlon ou des voies de communications inter- et intracellulaires.

\subsection{Motricité intestinale}

L'absorption des nutriments, de l'eau et des électrolytes ainsi que le transit du bol alimentaire sont en permanence favorisés par des contractions segmentaires et des contractions péristaltiques. Dans les phénomènes de diarrhée, deux cas peuvent se présenter : soit aucune contraction n'est présente au niveau du tube digestif, soit il y a des contractions propulsives intensives. Une exposition aux rayonnements ionisants peut moduler la motricité intestinale et par conséquent le transit du bol alimentaire en agissant, soit directement sur le muscle lisse, soit indirectement par l'intermédiaire des agents libérés par les nerfs ou des cellules sous-jacentes. De plus, d'autres agents libérés à distance et véhiculés dans le sang peuvent aussi modifier l'activité des cellules musculaires.

Chez l'homme, une augmentation du transit total n'a été montrée que pendant la première semaine qui suit l'irradiation de la sphère abdomino-pelvienne pour raisons thérapeutiques (Yeoh et al., 1993a). Dans d'autres études, notamment chez le chien, des augmentations de l'activité propulsive ont été rapportées (Erikson et al., 1994) en accord avec la présence de diarrhée. Chez le rat, Picard et al. ont montré que la motricité intestinale et colique, observée grâce à l'enregistrement de l'activité électrique, était inhibée (diminution des contractions) dès 24 heures après l'irradiation (Picard et al., 2001). Cette inhibition est très marquée trois jours après l'irradiation avec une désorganisation totale du profil moteur au niveau de l'intestin concomitant à une accélération du transit intestinal et à l'apparition de la diarrhée. Ces altérations de la motricité intestinale se poursuivent jusqu'au $7^{\text {e jour. }}$ L'absence des contractions ne permet plus la rétention des digesta au niveau de l'intestin et entraîne une augmentation du flux des contenus intestinaux. 
Ces altérations de la motricité intestinale pourraient aussi être la cause des crampes et des douleurs abdominales, souvent rencontrées chez les personnes irradiées.

\subsection{Absorption et sécrétion}

Une malabsorption des nutriments peut résulter d'une irradiation et être en partie responsable de la perte de poids souvent observée. Becciolini a montré, chez des patients ayant reçu une radiothérapie, une diminution de 25 à $30 \%$ de l'absorption des lipides, de la vitamine B12 et des hydrates de carbone. L'absorption des acides aminés est moins atténuée (Becciolini, 1987). En effet, chez le rat, Cheeseman et al. (1985) ont observé une augmentation de l'activité des systèmes de transport des acides aminés malgré une réduction de leur nombre. Sur le plan clinique pendant la phase aiguë, les modifications du transport de l'eau et des électrolytes sont les plus urgentes à gérer car le maintien de la balance hydrominérale ne peut pas être assuré par des perfusions pendant de longues périodes.

De nombreuses études ont été faites sur les mouvements d'eau et d'électrolytes après irradiation. Pour des doses n'entraînant pas le syndrome gastro-intestinal une augmentation de la sécrétion du chlore (estimée par la mesure du courant de court circuit de l'intestin grêle isolé) a été montrée chez plusieurs espèces (lapin, rat, furet) pendant les premières 24 à 48 heures suivant l'irradiation (Gunter-Smith, 1986 ; MacNaughton et al., 1994, 1997). Ces mesures sont en accord avec une perte d'eau par l'intestin grêle. L'absorption d'eau et d'électrolytes est également atténuée 12 heures après une exposition à des rayons X de 15 Gy (Curran et al., 1960). Ces observations montrent que des altérations fonctionnelles de l'intestin apparaissent avant la dénudation de la muqueuse.

L'augmentation du flux hydrique dans l'intestin grêle n'est pas toujours suivie par une diarrhée grâce à la "capacité de réserve » du côlon. Cependant, quand cette capacité est dépassée et/ou que le côlon est endommagé, les diarrhées apparaissent. Le côlon a longtemps été considéré comme moins radiosensible que l'intestin grêle. Cette relative « radio-tolérance » colique pourrait s'expliquer par un temps de renouvellement épithélial un peu plus long que celui de l'intestin grêle (Potten, 1990). Néanmoins, Wilson en 1959 a décrit les atteintes coliques comme étant déterminantes dans les décès radio-induits (Wilson, 1959). D'autre part, après des irradiations thérapeutiques localisées (cancers de la prostate, de l'utérus...) le côlon pourrait être impliqué dans les effets secondaires de l'exposition car il est quasi systématiquement dans le champs d'irradiation.

En ce qui concerne les altérations fonctionnelles du côlon, les études in vivo chez le rat ont montré une diminution de l'absorption d'eau et d'électrolytes (Empey et al., 1992 ; Weiber et al., 1993 ; Dublineau et al., 1998) pendant la phase 
aiguë sans évidence histologique de modifications profondes de la muqueuse colique. D'autres études in vivo, chez le rat, ont montré l'effet d'une augmentation de la composante neutron dans le cas d'une fluence mixte neutron/gamma (dose totale $=3,8 \mathrm{~Gy}$ ). Le flux hydrique absorptif est d'autant plus diminué que la composante neutronique est augmentée 4 jours après irradiation (François et al., 1999). Parallèlement, les anomalies morphologiques (perte des cryptes, diminution de l'épaisseur de la muqueuse, sécrétion du mucus quasiment nulle) sont très marquées lorsque la composante neutronique est plus forte. Ces résultats comparés à ceux obtenus avec un rayonnement gamma pour la même fonction colique ont permis d'estimer un EBR d'environ 2.

\subsection{Modifications des régulations et de l'environnement}

Les modifications radio-induites du fonctionnement du système digestif peuvent provenir des altérations de la libération et/ou du comportement des facteurs de régulation. Les différents types cellulaires impliqués dans la libération de ces facteurs sont les cellules endocrines, localisées dans la muqueuse, les cellules immunocompétentes, situées dans la sous-muqueuse ou entre les cellules épithéliales, et enfin les terminaisons nerveuses du système nerveux entérique (SNE). Les facteurs luminaux jouent également un rôle, principalement sur la barrière intestinale en agissant sur différents types cellulaires. Ils peuvent donc participer à la réponse de l'épithélium, des couches musculaires ou des cellules immunocompétentes. Parmi ces facteurs luminaux, les acides biliaires peuvent contribuer aux atteintes intestinales et coliques.

Chez des patients irradiés pour des raisons thérapeutiques, dans un champ abdominal, Yeoh et al. ont montré une malabsorption des acides biliaires concomitantes à une augmentation du transit dans l'intestin grêle et à une augmentation du nombre de selles (Yeoh et al., 1993a, 1993b). Ces agents, en particulier les acides biliaires hydrophobes, peuvent avoir des effets agressifs sur la muqueuse intestinale et/ou colique, stimuler la sécrétion de chlore et provoquer la diarrhée. Chez le rat, il a été observé que la teneur en acides biliaires dans les fèces augmente en fonction de la dose d'irradiation. Cette augmentation est significative dès le lendemain de l'irradiation pour une dose supérieure à $4 \mathrm{~Gy}$. Les modifications de la composition du pool des acides biliaires arrivant dans l'intestin proximal sont également dépendantes de la dose d'irradiation, 3 jours après exposition. Le rapport [acides biliaires dihydroxylés/acides biliaires trihydroxylés] qui peut être considéré comme un index de l'agressivité du «pool » biliaire vis-àvis de l'intestin est fortement augmenté pour des doses supérieures à 6 Gy chez le rat. Des résultats similaires ont également été obtenus chez le porc après l'exposition gamma seule ou mixte gamma/neutron (Scanff et al., 1997, 1999). 
Il est généralement admis qu'une réponse inflammatoire est générée suite à une exposition aux rayonnements ionisants. Chez des patients irradiés pour des raisons thérapeutiques, une augmentation du taux de prostaglandine E2 $\left(\mathrm{PGE}_{2}\right)$ et de leucotriène-B4 $\left(\mathrm{LTB}_{4}\right)$, deux agents souvent associés à des réponses inflammatoires, a été retrouvée dans les dialysats rectaux (Cole et al., 1993). D'autres études ont montré une augmentation de la capacité de synthèse de $\mathrm{PGE}_{2}$ dans le côlon chez le rat après une irradiation abdominale (Monti et al., 1998). Cet effet est d'autant plus fort que la dose d'irradiation est élevée. L'étude cinétique a montré que les modifications de la capacité de synthèse de $\mathrm{PGE}_{2}$ sont plus importantes 4 jours après l'irradiation et que l'on retrouve des valeurs normales 7 jours après l'exposition. Ces résultats montrent que l'irradiation entraîne une libération accrue de prostaglandines, susceptibles d'amplifier les phénomènes sécrétoires et d'altérer les fonctions motrices au niveau de ce segment intestinal.

D'autres médiateurs peuvent être libérés par plusieurs types cellulaires, qui proviennent principalement des cellules résidentes sur l'épithélium intestinal. C'est le cas de la neurotensine, un peptide retrouvé dans les cellules $\mathrm{N}$. La neurotensine qui agit (via sa fixation sur des récepteurs spécifiques) sur les transports d'électrolytes en inhibant l'absorption du chlore ainsi que sur la contractilité musculaire est un des médiateurs pouvant contribuer à la genèse de la diarrhée radio-induite. Il a été observé chez le rat 3 jours après une irradiation totale de 6 Gy $(\gamma)$ que les taux plasmatiques et tissulaires ainsi que le nombre de cellules $\mathrm{N}$ sont augmentés. Ces variations tissulaires sont accompagnées d'une modification de la réponse contractile du côlon isolé, d'une augmentation du nombre total de sites de liaison et de l'apparition d'un second type de récepteur sur les cellules musculaires (Linard et al., 1997 ; Lehy et al., 1997).

Ainsi, l'irradiation totale à une dose inférieure à celle qui déclenche le syndrome gastro-intestinal, modifie les agents luminaux ainsi que les médiateurs libérés par l'épithélium et les cellules immunocompétentes. De plus, les modifications structurales de l'épithélium et des cellules de soutien, plus particulièrement les myofibroblastes, peuvent avoir un rôle important. Chez des patients irradiés au niveau de la sphère abdominale, Wiernick et Perrins ont observé une diminution du nombre de cellules épithéliales ainsi qu'une diminution du nombre de myofibroblastes (Wiernick et Perrin, 1975). À la fin du traitement, le nombre des myofibroblastes est toujours réduit tandis que celui des cellules épithéliales est redevenu normal. Des études récentes, chez le rat irradié, ont montré que la perméabilité de la muqueuse colique est augmentée, associée à une désorganisation concomitante de la structure des connections inter- et intracellulaires. Ces altérations apparaissent au niveau des cryptes et des myofibroblastes (cellules de l'espace péricryptal). Des modifications des taux de certaines protéines participant aux interactions intercellulaires (E-cadhérine, $\beta$-caténine) et de facteurs 
mitochondriaux (cytochrome c) et cytoplasmiques (caspase-3) participant à leur dégradation ont été trouvés. L'ensemble de ces résultats suggère que l'irradiation induit une cascade de réactions commençant par une libération de facteurs mitochondriaux qui vont provoquer une dégradation des interactions intercellulaires conduisant à une perte de cohésion de la barrière intestinale et à des perturbations de la capacité d'absorption des cryptes coliques (Thiagarajah et al., 2000).

\section{Traitements}

À l'heure actuelle, les traitements des atteintes digestives radio-induites sont symptomatiques. Pour les vomissements, les inhibiteurs des récepteurs de la sérotonine de type 3 (granisétron, ondansétron) sont efficaces. Ainsi, nous avons montré chez le rat qu'un traitement de granisétron après une irradiation abdominale de 10 Gy réduisait la diminution radio-induite de la motricité et améliorait l'absorption d'eau dans le côlon. Toutes ces mesures étaient en accord avec le profil clinique, puisque les animaux traités au granisétron n'avaient pas de diarrhée (Picard et al., 2002). Néanmoins, il reste à prouver leur efficacité sur la diarrhée réflexe précoce. Le lopéramide, qui a des propriétés anti-motrice et antisécrétoire, est souvent employé pour traiter la diarrhée, soit pendant la phase précoce soit pendant la phase «manifeste ». Cependant ces molécules ne sont pas efficaces contre la perte de l'intégrité de la barrière intestinale. Deux approches sont nécessaires :

(1) limiter les agressions par les agents luminaux tels que les bactéries et/ou leurs toxines, les acides biliaires, les antigènes ainsi que par les médiateurs libérés par les nerfs, les cellules de l'épithélium et celles de l'environnement sousjacent (myofibroblastes...) ;

(2) stimuler la régénération des cellules de la barrière épithéliale et celles de l'environnement et/ou retarder la desquamation des cellules de la muqueuse intestinale.

Parmi les nouvelles approches, utilisées en cas de maladie intestinale inflammatoire ainsi qu'avant irradiation totale (pré-traitement avant une greffe de moelle osseuse), le traitement avec une cytokine anti-inflammatoire, l'Interleukine-11, semble être prometteur. En effet, cet agent est reconnu pour avoir des effets bénéfiques sur la restauration de la barrière intestinale, notamment sur la réduction de la translocation bactérienne après les agressions chimiques ou une exposition aux rayonnements ionisants et dans le cas de la réaction du greffon contre l'hôte (GvHD) (Orazi et al., 1996). Une autre molécule utilisée pour protéger la muqueuse intestinale est le Kératinocyte Growth Factor (KGF) qui est produit et sécrété par les cellules sous-jacentes de l'épithélium (myofibroblastes). Le KGF est un facteur de croissance agissant sur la muqueuse intestinale. II semble 
avoir des effets bénéfiques contre différents types d'agressions telles que la radioou chimiothérapie, le GvHD et la translocation bactérienne. De plus il stimule le glutathion (molécule anti-oxydante) et l'intestinal trefoil factor. Un traitement avec le KGF provoque une prolifération de tous les types cellulaires de l'épithélium intestinal (Farrell et al., 1998). D'autres agents ont été testés contre les effets de l'irradiation, la plupart ayant été donné avant l'exposition aux rayonnements. L'insulin-like growth factor (IGF-1), le transforming growth factor- $\beta$ (TGF- $\beta$ ), le fibroblast growth factor (FGF) semblent efficaces en tant que radio-protecteurs, leur efficacité lorsqu'ils sont administrés après l'exposition restant à prouver (Sharp, 1994). Néanmoins, dans l'étude effectuée par Paris et al. un traitement au FGF après irradiation semble avoir un effet bénéfique contre les atteintes de l'endothélium intestinal (Paris et al., 2001).

L'administration de nutriments administrés par voie entérale, par exemple un régime riche en glutamine, pourrait également avoir des effets bénéfiques (Chary et al., 1984). La cholestyramine est parfois utilisée en tant que piège des acides biliaires qui peuvent avoir des effets néfastes sur une muqueuse déjà endommagée (Huang et al., 2000).

À l'heure actuelle les approches thérapeutiques des atteintes digestives radioinduites restent symptomatiques puisqu'elles inhibent l'action des médiateurs libérés ou celle des agents luminaux. L'utilisation de facteurs stimulant la croissance cellulaire semble prometteuse. Dans l'avenir des transplantations intestinales ou l'apport de cellules intestinales (cellules souches intestinales et/ou de l'environnement) pourraient être envisagés.

\section{RÉFÉRENCES}

Baranov A.E., Guskova A.K. (1990) The Medical Basis for Radiation Accident Preparedness II, pp. 79-86. Elsevier, New York.

Baranov A.E. (1994) Adv. Biosci. 94, 281-293.

Becciolini A. (1987) Relative radiosensitivities of the small and large intestine. Adv. Radiat. Biol. 12, $83-128$.

Chary S., Thomson D.H. (1984) A clinical trial evaluating cholestyramine to prevent diarrhea in patients maintained on low-fat diets during pelvic radiation therapy. Int. J. Radiat. Oncol. Biol. Phys. 10, 1885-1890.

Cheeseman C.L., Thomson A.B.R., Walker K. (1985) The effects of abdominal irradiation on intestinal transport in the rat as assessed with isolated epithelial cells. Radiat. Res. 101, 131-143.

Cole A.T., Slater K., Sokal M., Hawkey C.J. (1993) In vivo rectal inflammatory mediator changes with radiotherapy to the pelvis. Gut 34, 1210-1214.

Curran P.F., Webster E.W., Housepain J.A. (1960) The effect of X-irradiation on sodium and water transport in rat ileum. Radiat. Res. 13, 360-380.

Deitch E.A., Ma L., Ma J.W., Berg R.D. (1989) Lethal burn-induced bacterial translocation: role of genetic resistance. J. Trauma. 29, 1480-1487. 


\section{N.M. GRIFFITHS et al.}

Dublineau I., Ksas B., Aigueperse J., Gourmelon P., Griffiths N.M. (1998) In vivo alterations of fluid and electrolyte fluxes in the rat colon by gamma irradiation. Dig. Dis. Sci. 43, 652-662.

Empey L.R., Papp J.D., Jewell L.D., Fedorak R.N. (1992) Mucosal protective effects of vitamin E and misoprostol during acute radiation-induced enteritis in rats. Dig. Dis. Sci. 37, 205-214.

Erikson B.A., Otterson M.F., Moulder J.E., Sarna S.K. (1994) Altered motility causes the early gastrointestinal toxicity of irradiation. Int. J. Radiat. Oncol. Biol. Phys. 28, 905-912.

Farrell C.L., Bready J.V., Rex K.L., Chen J.N., DiPalma C.R., Whitcomb K.L., Yin S., Hill D.C., Wiemann B., Starnes C.O., Havill A.M., Lu Z.N., Aukerman S.L., Pierce G.F., Thomason A., Potten C.S., Ulich T.R., Lacey D.L. (1998) Keratinocyte growth factor protects mice from chemotherapy and radiation-induced gastrointestinal injury and mortality. Cancer. Res. 58, 933-939.

François A., Dublineau I., Lebrun F., Ksas B., Griffiths N.M. (1999) Modified absorptive and secretory processes in the rat colon following neutron irradiation: in vivo and in vitro studies. Radiat. Res. 151, 468-478.

Gunter-Smith P.J. (1986) Gamma irradiation affects active electrolyte transport by rabbit ileum. Basal Na and Cl transport. Am. J. Physiol. 250, G540-G545.

Hendry J.H., Potten C.S. Roberts N.P. (1983) The gastrointestinal syndrome and mucosal clonogenic cells: relationships between target cell sensitivities, LD50 and cell survival and their modification by antibiotics. Radiat. Res. 96, 100-112.

Hill G.R., Crawford J.M., Cooke K.J., Brinson Y.S., Pan L., Ferrara J.L.M. (1997) Total body irradiation and acute graft-versus-host disease: the role of gastrointestinal damage and inflammatory cytokines. Blood 90, 3204-3213.

Huang E.Y., Leung S.W., Wang C.J., Chen H.C., Sun L.M., Fang F.M., Yeh S.A., Hsu H.C., Hsiung C.Y. (2000) Oral glutamine to alleviate radiation-induced oral mucositis: a pilot randomized trial. Int. J. Radiat. Oncol. Biol. Phys. 46, 535-539.

IAEA (1988) The Radiological Accident in Goiânia, Vienna, Austria, pp. 41-50.

IAEA (1993) The Radiological Accident in Soreq, Vienna, Austria, pp. 31-70.

IAEA (1996) The Radiological Accident in at the Irradiation Facility in Nesvizh, Vienna, Austria, pp. 46-65.

Lehy T., Dessirier V., Bado A., Griffiths N.M., Linard C. (1997) Exposure to ionizing radiation modifies circulating gastrin levels and gastrointestinal endocrine cell densities in the rat. Int. $J$. Radiat. Biol. 73, 331-340.

Letschert J.G., Lebesque J.V., Aleman B.M., Bosset J.F., Horiot J.C., Bartelink H., Cionini L., Hamers J.P., Leer J.W., van-Glabbeke M. (1994) The volume effect in radiation-related late small bowel complications: results of a clinical study of the EORTC Radiotherapy Cooperative Group in patients treated for rectal carcinoma. Radiother. Oncol. 32, 116-123.

Linard C., Griffiths N.M., Esposito V., Aigueperse J., Gourmelon P. (1997) Changes in gut neurotensin and modified colonic motility following whole-body irradiation in rat. Int. J. Radiat. Biol. 71, 581-588.

MacNaughton W.K., Leach K.E., Prudhomme-Lalonde L., Ho W., Sharkey K. (1994) Exposure to ionizing radiation increases responsiveness to neural secretory stimuli in the ferret jejunum in vitro. Gastroenterol. 106, 324-335.

MacNaughton W.K., Leach K.E., Prudhomme-Lalonde L., Harding R.K. (1997) Ionizing radiation reduces neurally-evoked electrolyte transport in rat ileum through a mast cell-dependent mechanism. Int. J. Radiat. Biol. 72, 219-226.

Maejima K., Deitch E.A., Berg R. (1984) Promotion by burn stress of the translocation of bacteria from the gastrointestinal tracts of mice. Arch. Surg. 119, 166-17.2.

Monti P., Ksas B., Griffiths N.M. (1998) European Radiation Research'98, October 1998, Cápri, Italy.

Moore F.A. (1999) Role of gut hypoperfusion in the development of systemic inflammatory response syndrome (SIRS) and multiple organ failure (MOF). Am. J. Surg. 178, 449-453. 
Orazi A., Du X., Yang Z., Kashai M., Williams D.A. (1996) Interleukin-11 prevents apoptosis and accelerates recovery of small intestinal mucosa in mice treated with combined chemotherapy and radiation. Lab. Invest. 75, 33-42.

Paris F., Fuks Z., Kang A., Capodieci P., Juan G., Ehleiter D., Haimowitz-Friedman A., Cordon-Cardo C., Kolesnick R. (2001) Endothelial apoptosis as the primary lesion initiating intestinal radiation damage in mice. Science 293, 293-297.

Picard C., Linard C., Wysocki J., Griffiths N.M. (2001) Role of intestinal MMC in diarrhoea induced by abdominal irradiation in rats. Neurogastroenterol. Motil. 13, 19-26.

Picard C., Wysocki J., Griffiths N.M, Fioramonti J. (2002) Effect of granisetron treatment on radiationinduced alterations of colonic motility and fluid absorption in rats. Aliment. Pharmacol. Ther, 16, 623-631.

Potten C.S. (1990) A comprehensive study of the radiobiological response of the murine (BDF1) intestine. Int. J. Radiat. Biol. 58, 925-973.

Quastler H. (1956) The nature of intestinal radiation death. Radiat. Res. 4, 303-320.

Scanff P., Grison S., Monti P., Joubert C., Griffiths N.M., Gourmelon P. (1997) Whole-body irradiation modifies bile composition in the pig. Radiat. Res. 148, 175-180.

Scanff P., Monti P., Joubert C., Grison S., Gourmelon P., Griffiths N.M. (1999) Mixed field neutrons and gamma photons modify bile acid profiles in the pig. Int. J. Radiat. Biol. 75, 209-216.

Sharp J.G. (1994) The potential use of cytokines to ameliorate the effects of gastrointestinal radiation injury. Adv. Biosci. 94, 217-231.

Spitzer T.R. (1995) Radiation and the Gastrointestinal Tract, pp. 3-19. CRC, Boca Raton.

Thiagarajah J., Gourmelon P., Griffiths N.M., Lebrun F., Naftalin R.J., Pedley K.C. (2000) Radiation induced cy tochrome $\mathrm{c}$ release causes loss of rat colonic fluid absorption by damage to crypts and pericryptal myofibroblasts. Gut 47, 675-684.

Weiber S., Bjelkengren G., Rank F., Jibom H., Zederfelt B. (1993) Radiation effects in the colon. Acta. Oncol. 32, 565-569.

Wiernick G., Perrins D. (1975) The radiosensitivity of a mesenchymal tissue. The pericryptal fibroblast sheath in the human rectal mucosa. Br. J. Radiol. 48, 382-389.

Wilson S.G. (1959) Radiation-induced gastrointestinal death in the monkey. Amer. J. Pathol. 35, 1233 1251.

Yeoh E, Horowitz M, Russo A, Muecke T, Robb T., Maddox A., Chatterton BA. (1993a) Effect of pelvic irradiation on gastrointestinal function: a prospective longitudinal study. Am. J. Med. $\mathbf{9 5}$, 397-406.

Yeoh E., Horowitz M., Russo A., Muecke T., Ahmad A., Chatterton B.A. (1993b) A retrospective study of the effects of pelvic irradiation for carcinoma of the cervix on gastrointestinal function. Int. J. Radiat. Oncol. Biol. Phys. 26, 229-237. 